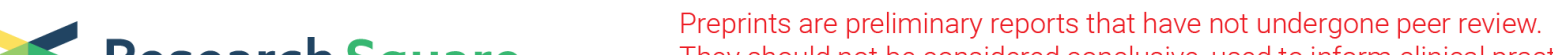 Research Square They should not be considered conclusive, used to inform clinical practice, or referenced by the media as validated information.
}

\section{Mental Health Burden of Patients With Diabetes Before And After The Outbreak of COVID-19: Predictors of Mental Health Impairment}

\section{Sheila Moradian ( $\sim$ Sheila.moradian@uni-due.de)}

Clinic for Psychosomatic Medicine and Psychotherapy, University of Duisburg-Essen, LVR University Hospital

\section{Martin Teufel}

Clinic for Psychosomatic Medicine and Psychotherapy, University of Duisburg-Essen, LVR University Hospital

\section{Lisa Jahre}

Clinic for Psychosomatic Medicine and Psychotherapy, University of Duisburg-Essen, LVR University Hospital

\section{Venja Musche}

Clinic for Psychosomatic Medicine and Psychotherapy, University of Duisburg-Essen, LVR University Hospital

\section{Madeleine Fink}

Clinic for Psychosomatic Medicine and Psychotherapy, University of Duisburg-Essen, LVR University Hospital

\section{Hannah Kohler}

Clinic for Psychosomatic Medicine and Psychotherapy, University of Duisburg-Essen, LVR University Hospital

\section{Adam Schweda}

Clinic for Psychosomatic Medicine and Psychotherapy, University of Duisburg-Essen, LVR University Hospital

\section{Benjamin Weismüller}

Clinic for Psychosomatic Medicine and Psychotherapy, University of Duisburg-Essen, LVR University Hospital

\section{Nora Dörrie}

Clinic for Psychosomatic Medicine and Psychotherapy, University of Duisburg-Essen, LVR University Hospital

\section{Susanne Tan}

Department of Endocrinology, Diabetes and Metabolism, University of Duisburg-Essen, University Hospital Essen

\section{Eva-Maria Skoda}


Clinic for Psychosomatic Medicine and Psychotherapy, University of Duisburg-Essen, LVR University Hospital

\section{Alexander Bäuerle}

Clinic for Psychosomatic Medicine and Psychotherapy, University of Duisburg-Essen, LVR University Hospital

\section{Research Article}

Keywords: mental health, COVID-19, diabetes, changes in mental health, anxiety, depression

Posted Date: June 10th, 2021

DOl: https://doi.org/10.21203/rs.3.rs-506228/v1

License: (c) (1) This work is licensed under a Creative Commons Attribution 4.0 International License. Read Full License 


\section{Abstract}

Background: The COVID-19 pandemic is affecting people's mental health worldwide. Patients with diabetes are at risk for a severe course when infected with SARS-CoV-2. The present study aims to retrospectively examine mental health changes in patients with diabetes in Germany before and after the COVID-19 outbreak, and to furthermore explore potential predictors of such changes.

Methods: Over the course of eight weeks in early summer 2020, 253 individuals diagnosed with diabetes participated in an online cross-sectional study. Participants completed an anonymous survey including demographics, depression (PHQ-2) and generalized anxiety symptoms (GAD-2), distress (DT), and health status (EQ-5D-3L). In addition, all instruments used were adjusted to measure participants' mental health and health status before the COVID-19 outbreak. Additionally examined factors were COVID-19-related fear, trust in governmental actions to face the pandemic, and the subjective level of information about COVID-19.

Results: This study shows a significant increase in prevalence of depression symptoms, generalized anxiety symptoms and distress, as well as significantly decreased health statuses in diabetes patients after the COVID-19 outbreak. Increased depression symptoms, generalized anxiety symptoms and distress were predicted by COVID-19-related fear, whereas trust in governmental actions to face COVID-19 predicted higher depression symptoms.

Conclusions: The results indicate a negative impact of the COVID-19 outbreak on mental health and health status in patients with diabetes. In order to improve the efficacy of psychological support strategies for diabetes patients during the pandemic, possible predictors of mental health impairment such as the aforementioned should be examined more thoroughly and addressed more openly.

\section{Background}

Ever since its outbreak in China, the newly identified disease termed COVID-19 (Corona Virus Disease-19) has spread rapidly through the whole world. The spread of the underlying novel virus SARS-CoV-2 (severe acute respiratory syndrome coronavirus type 2) was officially declared as a global pandemic on March 11th 2020 [1] and has impacted every nation with a few exceptions [2]. Over 131 million infections have been confirmed worldwide, entailing severe disease and almost 2.9 million deaths (until May 2021 [3]).

To reduce the transmission of this now infamous strain of corona virus, many countries announced several restrictions, as vaccinations and medications were still lacking in the beginning. These restrictions included, among others, the shutdown of public facilities, a partial prohibition of social gatherings, the restriction of entry and intensification of border controls, as well as the promotion of safety behaviour. The restrictions, while abrupt and unprecedented, were considered inevitable in order to prevent capacity overload in hospitals and to protect risk groups [4]. 
These changes in everyday-life, alarming mortality levels in some countries and related media coverage had a large impact, not only on public life and the economic development, but also on people's mental health $[5,6]$. Recently published international literature investigating the mental health of different populations in the context of the pandemic, showed elevated prevalence in depression, anxiety and stress symptoms [7-12]. These findings are in line with recent research from Germany, which points toward increased prevalence of generalized anxiety symptoms, depression symptoms, distress and COVID-19 related fear in the German population during the first lock-down [13]. Furthermore, depression, anxiety and distress symptoms in individuals with pre-existing mental illnesses appeared to have worsened considerably [14]. Thus, pre-existing mental illness appears to be a risk factor for psychological burden, as well as being female, at younger age, a student or unemployed $[15,16]$.

Other risk factors for psychological burden in the context of COVID-19 include pre-existing somatic diseases like diabetes, arterial hypertension, cardiovascular or respiratory disorders $[17,18]$. The preexisting medical conditions of several somatic diseases can lead to a more severe course of COVID-19 [19] and thus they were defined as high-risk morbidities for COVID-19 [20, 21]. Diabetes is a definite risk factor for a severe course of COVID-19 [22]. This chronic metabolic disease is characterized by increased levels of blood glucose, affects about 422 million people worldwide and is directly attributed to 1.6 million deaths each year [23]. Several studies reported diabetes patients to be two to three times more likely to need intensive care, when infected with COVID-19, compared to patients with less severe disease, as well as a higher mortality in diabetes patients with COVID-19 [24, 25]. In recent literature a two-way interaction between COVID-19 and diabetes is stated, in which COVID-19 leads to worsening of dysglycemia and diabetes increased severity in COVID-19 courses [26]. Hence, patients appear to carry a double burden and need to be very careful to follow safety behaviour such as hand-washing or physical distancing [27] in order to minimize the possibility of infection and a severe course of COVID-19. A recent study showed that patients with diabetes reported higher COVID-19-related fear and more safety behaviour than matched controls [28]. Moreover, Joenson et al. [29] found that diabetes patients worried about not being able to manage their illness and treatment if infected with COVID-19.

Consequently, this increased risk for developing a severe course of COVID-19 because of a chronic somatic disease aggravates psychological burden due to COVID-19 [11, 30]. In addition to this pandemic stress, individuals with chronic diseases such as diabetes already have an increased psychological burden due to their medical condition [31]. The everyday-life of diabetes patients is characterized by challenges and complications resulting in considerable emotional distress [32], which can lead to a higher risk of mental disorders: Several studies showed higher prevalence of mental health issues in individuals with diabetes compared to individuals without diabetes [33], including depression and anxiety disorders [34-36]. Importantly, depression and anxiety symptoms may be associated with lower treatment adherence, which can lead to worse glycemic control and, ultimately, adverse diabetes outcomes [37]. As pre-existing mental health illness appears to be a risk factor for psychological burden [14], it can be assumed that patients with diabetes suffer from various stress factors during the COVID-19 pandemic. 
Thus, the COVID-19 pandemic poses a particular challenge to patients with diabetes by adding uncertainty and distress to an already stressful pre-existing condition. In order to provide adequate support for patients with diabetes it is important to understand how the COVID-19 outbreak affects them and if there are risk and protective factors. In recent investigations COVID-19-related fear, pre-existing mental illness, trust in governmental actions and the subjective level of information appeared to predict mental health impairment $[13,38]$. Due to the sudden emergence of the pandemic, mental health and health status can only be measured through patients' retrospective assessments. As patients with diabetes suffer from various stress factors, such as an objectively increased risk of a severe course of COVID-19 and pre-existing psychological burden, their mental health and health status might be impaired since the outbreak of COVID-19. We expect the aforementioned factors to negatively impact mental health and health status of patients with diabetes.

The increasing relevance of this issue is mirrored by the rising number of publications on the subject of mental health changes due to the COVID-19 pandemic. Rather than focusing on the general health effects of COVID-19 on a population, since several representative studies have already been conducted, the aim of this study was to examine depression symptoms, generalized anxiety symptoms, distress and health status after and, retrospectively, before the outbreak of COVID-19 in individuals with diabetes.

Furthermore, we aim to investigate predictors of health changes in patients with diabetes during the pandemic, in order to provide relevant insight to preventative and acute mental health services for vulnerable groups.

\section{Methods}

\subsection{Procedure and Participants}

The cross-sectional study was conducted over eight weeks in early summer of 2020 after the outbreak of COVID-19 in Germany. Data from 253 participants with diabetes was collected via an online survey. Distribution took place through diabetes-centered online channels (e.g. online newspaper) and social media channels (e.g. Facebook), as well as print media. Eligibility requirements for participants was a diagnosis of diabetes mellitus type 1, type 2 or other specific diabetes, age $\geq 18$ years, good command of the German language, and internet access. Electronic informed consent was obtained before participants started the online survey. Study participation was anonymous, voluntary, and could be terminated at any time without any negative consequences for the participant. The study was approved by the Ethics Committees of the University Hospital Essen (20-9307-BO).

\subsection{Measures}

The survey was composed of self-generated items regarding socio-demographic data, medical details and attitudes towards COVID-19, as well as validated clinical instruments and their adapted versions, assessing mental and general health variables. 
Socio-demographic and medical details. Participants were asked to give information on gender, age, marital status, education, employment and population of their residence. Diagnosed diabetes type, quality of diabetes control, accompanying somatic illnesses and mental illness were also assessed.

Patient Health Questionnaire-2 (PHQ-2). The PHQ-2 consists of two items assessing depressive symptoms over the past two week on a four-point Likert scale ( $0=$ "never" to $3=$ "nearly every day" [39]). The cut-off for major depression symptoms is a sum score of $\geq 3$ points. Internal consistency was high, with Cronbach's $\mathrm{a}=.842$.

Generalized Anxiety Disorder Scale-2 (GAD-2). The GAD-2 is composed of two items screening for generalized anxiety symptoms over the past two week on a four-point Likert scale ( $0=$ "never" to $3=$ "nearly every day" $[40,41])$. Sum scores of $\geq 3$ indicate severe generalized anxiety symptoms. Internal consistency was high, with Cronbach's $\mathrm{a}=.835$.

Distress Thermometer (DT). The visual analogue scale of the Distress Thermometer was used to measure distress in the past week ( $0=$ "no distress" to $10=$ "extreme distress" [42]). The cut off for elevated distress is a score of $\geq 4$ points.

European Quality of Life 5 Dimensions 3 Level questionnaire (EQ-5D-3L). The visual analogue scale EQ$5 \mathrm{D}-3 \mathrm{~L}$ was used to assess health status $(0=$ "the worst health you can imagine" to $100=$ "the best health you can imagine" [43])

Retrospective assessment of mental health and health status. PHQ-2, GAD-2, DT and EQ-5D-3L were adapted to retrospectively assess mental health and health status before the outbreak of the COVID-19 pandemic (e.g. "Before the outbreak of COVID-19 (corona virus), how often have you been bothered by any of the following problems?"). Internal consistency for PHQ-2 (pre) and GAD-2 (pre) was high, with Cronbach's $\mathrm{a}=.815$ and Cronbach's $\mathrm{a}=.823$, respectively.

COVID-19-related fear was assessed by the self-generated item "I worry about COVID-19", scaled on a seven-point Likert scale ( 1 = "very low" to 7 = "extremely high").

Subjective level of information about COVID-19 was assessed by self-generated items "I feel informed about COVID-19", "I understand the health authorities' advice regarding COVID-19" and "I feel informed about measures to avoid an infection with COVID-19", scaled on a seven-point Likert scale ( $1=$ "complete disagreement" to 7 = "complete agreement"). The scale showed acceptable internal consistency with Cronbach's $\mathrm{a}=.788$.

Trust in government was assessed by the self-generated items "I think all governmental measures are being taken to combat COVID-19", "I have confidence in the governmental system in Germany" and "I think Germany is well prepared to face COVID-19", scaled on a seven-point Likert scale ( $1=$ "complete disagreement" to 7 = "complete agreement"). Internal consistency was high, with Cronbach's a $=.836$.

\subsection{Statistical Analyses}


Statistical analyses were realized using the statistical program for social sciences SPSS version 26 (IBM, New York, NY, USA) and R (4.0.3). For PHQ-2 and GAD-2 sum scores were calculated, as well as mean scores for the scales trust in government and subjective level of information. Normality of the sampling distribution was assumed due to large sample sizes [44]. For sociodemographic data, measures of general and mental health, as well as COVID-19-related scales descriptive statistics were conducted. Paired $t$-tests were applied to test for differences in mental health (PHQ-2, GAD-2, DT) and health status (EQ-5D-3L) before and after the outbreak of COVID-19 in patients with diabetes. Cohen's $d$ was used as effect size, with a $d$-value around $0.2,0.5$, and 0.8 being considered as small, medium-sized, and large effect, retrospectively. Differences in depression symptoms, generalized anxiety symptoms, distress, and health status acted as dependent variables for multiple regression models. Therefore difference values for PHQ-2, GAD-2, DT and EQ-5D-3L were calculated and included as dependent variables in the models, so that possible predictors for change could be identified. The predictors mental illness, COVID-19-related fear, trust in government and subjective level of information have been investigated as possible predictors in the general population [13] and in patients with cancer [38] before and were therefore investigated as possible predictors in our study. Homoscedasticity was given for GAD-2, DT and EQ5D-3L, as Breusch-Pagan tests indicated, with $p>0.05$. For this reason Huber-White standard errors were used. The level of significance was defined as $a=0.05$ (two-sided tests).

\section{Results}

\subsection{Sample Description}

The sample of 253 patients diagnosed with diabetes consists of 188 women (74.3\%) and 65 men (25.7\%) who were aged between 18 and 44 years (49.8\%) and 45 years and older (50.2\%). 169 participants reported to have type 1 (66.8\%), 74 type $2(29.2 \%)$ and 10 another specific diabetes mellitus (4.0\%). An overview of the sociodemographic and medical characteristics of the sample is presented in Table 1. 
Table 1

Sociodemographic and medical characteristics

\begin{tabular}{|c|c|c|}
\hline & $\mathbf{N}$ & $\%$ \\
\hline \multicolumn{3}{|l|}{ Sex } \\
\hline Female & 188 & 74.3 \\
\hline Male & 65 & 25.7 \\
\hline \multicolumn{3}{|l|}{ Age } \\
\hline$<45$ years & 126 & 49.8 \\
\hline$\geq 45$ years & 127 & 50.2 \\
\hline \multicolumn{3}{|l|}{ Marital status } \\
\hline Single & 64 & 25.3 \\
\hline Married & 117 & 46.2 \\
\hline In a relationship & 55 & 21.8 \\
\hline Divorced/separated & 14 & 5.5 \\
\hline Widowed & 3 & 1.2 \\
\hline \multicolumn{3}{|l|}{ Educational level } \\
\hline University education & 72 & 28.5 \\
\hline Higher education entrance qualification & 88 & 34.8 \\
\hline Higher secondary education & 55 & 21.7 \\
\hline Lower secondary education & 36 & 14.2 \\
\hline No degree & 2 & 0.8 \\
\hline \multicolumn{3}{|l|}{ Employment } \\
\hline In education & 15 & 5.9 \\
\hline Full employment & 102 & 40.3 \\
\hline Partial employment & 53 & 21.3 \\
\hline Not employed & 14 & 5.5 \\
\hline Retirement & 35 & 13.9 \\
\hline Sick leave & 13 & 5.1 \\
\hline Other & 20 & 7.9 \\
\hline
\end{tabular}




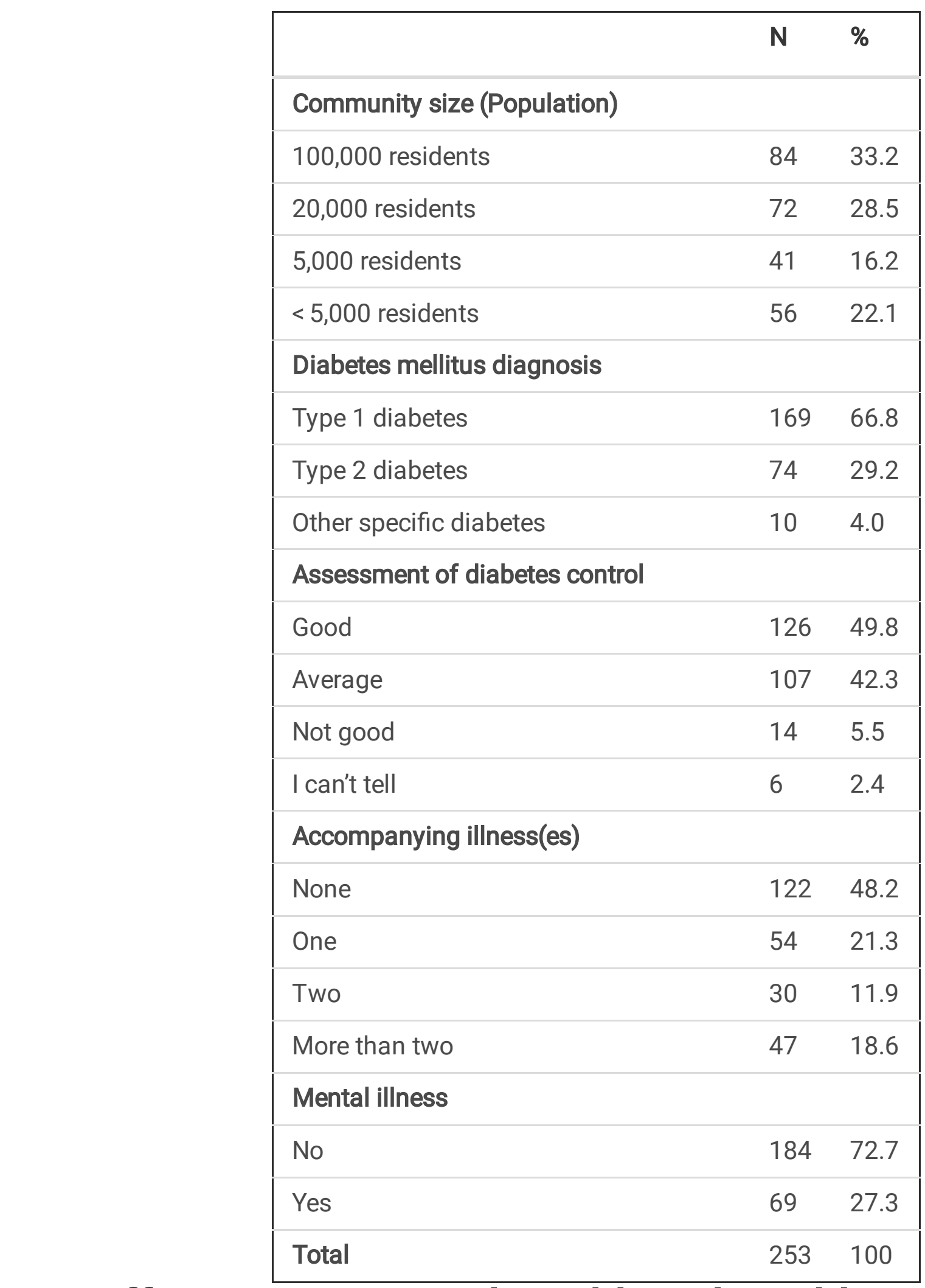

\subsection{Differences in Mental Health and Health Status Before and After the Outbreak of COVID-19}

The results of paired $t$-tests comparing mean values of PHQ-2, GAD-2, DT, and EQ-5D-3L before and after the outbreak of COVID-19 revealed a significant increase in depression symptoms, $t(252)=5.70, p<.001$, $d=0.185$, generalized anxiety symptoms, $t(252)=4.64, p<.001, d=0.263$, distress, $t(252)=7.06, p<.001$, 
$d=0.330$, and a decrease in health status, $t(252)=-3.51, p=.001, d=0.127$. The mean scores for each scale before and after the outbreak of COVID-19 are presented in Fig. 1.

\subsection{Prevalence of Depression Symptoms, Generalized Anxiety Symptoms, and Distress Before and After the COVID-19 Outbreak}

The prevalence of major depression symptoms increased from $11.9 \%$ before the COVID-19 outbreak to $21.3 \%$ after the outbreak. For severe generalized anxiety symptoms, an increase from $13.8-22.9 \%$ could be shown. There was an elevation in prevalence of elevated distress from $53.8 \%$ before the pandemic to $65.6 \%$ after the outbreak of COVID-19. For an overview, see Table 2.

Table 2

Prevalence of depression symptoms, generalized anxiety symptoms, and distress before and after the COVID-19 outbreak in patients with diabetes.

\begin{tabular}{|lll|}
\hline & $\begin{array}{l}\text { Before COVID-19 outbreak } \\
\text { N(\%) }\end{array}$ & $\begin{array}{l}\text { After COVID-19 outbreak } \\
\mathbf{N}(\%)\end{array}$ \\
\hline PHQ-2 & $223(88.1 \%)$ & $199(78.7 \%)$ \\
\hline$<3$ & $30(11.9 \%)$ & $54(21.3 \%)$ \\
\hline$\geq 3$ & $218(86.2 \%)$ & $195(77.1 \%)$ \\
\hline GAD-2 & $35(13.8 \%)$ & $58(22.9 \%)$ \\
\hline$<3$ & $117(46.2 \%)$ & $87(34.4 \%)$ \\
\hline$\geq 3$ & $136(53.8 \%)$ & $166(65.6 \%)$ \\
\hline DT & $253(100 \%)$ & $253(100 \%)$ \\
\hline$<4$ & & \\
\hline$\geq 4$ & & \\
\hline Total & & \\
\hline $\begin{array}{l}\text { Note: PHQ-2 = Patient Health Questionnaire-2, sum scores of } \geq 3 \text { indicate major depression } \\
\text { symptoms; GAD-2 = Generalized Anxiety Disorder Scale-2, sum scores of } \geq 3 \text { indicate severe } \\
\text { generalized anxiety symptoms; DT = Distress Thermometer, scores of } \geq 4 \text { indicate elevated distress. }\end{array}$ \\
\hline
\end{tabular}

\subsection{Predictors of Change in Mental Health and Health Status}

Tables 3-6 show the results of the univariate multiple regressions models predicting changes in mental and general health, consisting of difference values for depression symptoms (PHQ-2), generalized anxiety symptoms (GAD-2), and distress (DT), and health status, described by EQ-5D-3L. 
An increase in depression symptoms could be significantly predicted by COVID-19-related fear and trust in government, while mental illness and subjective level of information were not significant predictors. The model explains $9.4 \%$ of the variance.

COVID-19-related fear could also significantly explain an increase in generalized anxiety symptoms. Mental illness, subjective level of information and trust in government were not significant predictors, with an explained variance of $10.3 \%$.

Increase in distress was equally significantly predicted by COVID-19-related fear, while mental illness, subjective level of information and trust in government were non-significant predictors. The explained variance of the model is $6.2 \%$.

No significant predictors for deterioration in health status could be found.

Table 3

Regression Coefficients Predicting an Increase in PHQ-2.

\begin{tabular}{|lllll|}
\hline & ba & SEa & tvalue & $p$-value \\
\hline Intercept & -0.250 & 0.580 & -0.431 & 0.667 \\
\hline Mental illness & 0.266 & 0.168 & 1.582 & 0.115 \\
\hline COVID-19-related fear & 0.132 & 0.033 & 3.955 & $<0.001$ \\
\hline Subjective level of information & -0.176 & 0.092 & -1.913 & 0.057 \\
\hline Trust in government & 0.132 & 0.058 & 2.287 & 0.023 \\
\hline $\begin{array}{l}\text { Note. Dependent Variable: PHQ-2 (difference between before and after the COVID-19 outbreak). Total } \\
R^{2}=.094, F(4)=6.235, p<.001, n=253 . \text { aUnstandardized regression coefficients. SEa = Huber-White } \\
\text { standard errors. }\end{array}$ & & & \\
\hline
\end{tabular}

Table 4

Regression Coefficients Predicting an Increase in GAD-2.

\begin{tabular}{|lllll|}
\hline & ba & SEa & tvalue & $p$-value \\
\hline Intercept & -0.303 & 0.524 & -0.578 & 0.564 \\
\hline Mental illness & 0.068 & 0.179 & 0.380 & 0.704 \\
\hline COVID-19-related fear & 0.215 & 0.047 & 4.610 & $<0.001$ \\
\hline Subjective level of information & -0.077 & 0.092 & -0.851 & 0.396 \\
\hline Trust in government & 0.013 & 0.065 & 0.198 & 0.843 \\
\hline $\begin{array}{l}\text { Note. Dependent Variable: GAD-2 (difference between before and after the COVID-19 outbreak). Total } \\
R^{2}=.103, F(4)=7.566, p<.001, n=253 . \text { aUnstandardized regression coefficients. SEa = Huber-White } \\
\text { standard errors. }\end{array}$ & & & \\
\hline
\end{tabular}


Table 5

Regression Coefficients Predicting an Increase in Distress (DT).

\begin{tabular}{|lcccc|}
\hline & $b a$ & SEa & t-value & $p$-value \\
\hline Intercept & 0.532 & 1.608 & 0.498 & 0.628 \\
\hline Mental illness & 0.197 & 0.290 & 0.682 & 0.496 \\
\hline COVID-19-related fear & 0.253 & 0.072 & 3.500 & $<0.001$ \\
\hline Subjective level of information & -0.115 & 0.148 & -0.783 & 0.435 \\
\hline Trust in government & -0.089 & 0.099 & -0.905 & 0.367 \\
\hline $\begin{array}{l}\text { Note. Dependent Variable: DT (difference between before and after the COVID-19 outbreak). Total } R^{2} \\
=.062, F(4)=5.318,\end{array}$ & & & \\
\hline \begin{tabular}{l}
$p<.001, n=253$. aUnstandardized regression coefficients. SEa = Huber-White standard errors. \\
\hline
\end{tabular}
\end{tabular}

Table 6

Regression Coefficients Predicting a Deterioration in Health Status (EQ-5D-3L).

\begin{tabular}{|lllll|}
\hline & $b a$ & $S E a$ & $t$-value & $p$-value \\
\hline Intercept & -9.714 & 6.893 & -1.409 & 0.160 \\
\hline Mental illness & -3.078 & 2.349 & -1.310 & 0.191 \\
\hline COVID-19-related fear & -0.519 & 0.461 & -1.125 & 0.262 \\
\hline Subjective level of information & 1.757 & 0.982 & 1.789 & 0.075 \\
\hline Trust in government & 0.679 & 0.651 & 1.044 & 0.298 \\
\hline $\begin{array}{l}\text { Note. Dependent Variable: EQ-5D-3L (difference between before and after the COVID-19 outbreak). } \\
\text { Total } R^{2}=0.060,\end{array}$ & & & \\
\hline
\end{tabular}

$F(4)=3.518, p=.008, n=253$. aUnstandardized regression coefficients. SEa = Huber-White standard errors.

\section{Discussion}

\subsection{Principal Results}

Individuals with diabetes have an elevated risk for developing a severe course of COVID-19 [25] and suffer from pre-existing psychological burden due to their somatic disease [31]. To understand the extent and investigate predicting factors of these individuals' psychological burden is essential, as they suffer from various stress factors simultaneously and may need customised psychological support during the pandemic. The present study attempted to compare mental health states of individuals diagnosed with 
diabetes before and after the COVID-19 outbreak. The results show a significant increase in the prevalence of symptoms associated with a mental health burden such as depression, generalized anxiety and distress after the outbreak of COVID-19 compared to symptom prevalence before. The effect sizes for all compared symptom categories were small. Further data analyses indicated two factors predicting an increase in mental health burden since the COVID-19 outbreak: High COVID-19-related fear predicted an increase in depression symptoms, generalized anxiety symptoms, and distress, whereas trust in governmental actions to face COVID-19 predicted an increase in depression symptoms. No significant predictors for the reported deterioration in diabetes patients' health status could be found since the onset of COVID-19. Pre-existing mental illness and subjective level of information were no significant predictors of the increase in mental health burden or decrease in diabetes patients' health status.

The findings are in line with previous research from cross-sectional studies worldwide showing elevated mental health burden during the current pandemic $[7,9,38]$ and in individuals suffering from high-risk diseases [17] such as cancer [38]. Therefore, it appears that individuals with diabetes suffer strongly from psychological burden due to the pandemic. To understand the levels of stress exposure that go along with COVID-19 and a pre-existence of a somatic diseases such as diabetes is essential, as this group is already burdened with stress factors pertaining to its respective chronic somatic disease. Further stress factors due to the pandemic and the heightened risk of a severe course of COVID-19 might also increase the risk of developing mental disorders. In order to prevent such adverse effects, low-threshold access to psychological care for individuals diagnosed with diabetes seems more necessary than ever. Several approaches are already in use, such as telephone consultations or online support services for mentally burdened individuals during the ongoing pandemic $[45,46]$. As these services are designed primarily for the general population, specific support for patients with diabetes is still lacking.

This study shows that increased COVID-19-related fear is a risk factor for the increase of depression symptoms, generalized anxiety symptoms and distress, which is in accordance with previous research showing similar results in the general population in Germany [13] and in cancer patients [38]. Recent literature indicates that diabetes patients report overall higher COVID-19-related fear [28]. Moreover Kohler et al. [17] showed that the more high-risk diseases were reported, the higher COVID-19-related fear was. Thus, elevated levels of COVID-19-related fear might be seen as a reflection of diabetes patients' increased need for a sense of security. This hypothesis is supported by the fact that diabetes patients report more safety behaviour compared to individuals without diabetes [28]. In cognitive-behavioural psychotherapy it is assumed that safety behaviour arises out of a feeling of threat and can be considered as a coping strategy for anxiety [47]. Hence, COVID-19-related fear may be seen as a functional emotion which motivates a range of behaviours that reduce infection risk, but also burdens mental health to some extent. It might be crucial to educate patients with diabetes about these two sides of their COVID-19related fear in order to depathologize the emotion and, consequently, cope more adequately. However, the focus of current treatment of COVID-19 is on infection control, effective vaccine, and treatment cure rate $[48,49]$ and aspects of psychological impairment of high-risk groups such as patients with diabetes have yet to be thoroughly considered and addressed more openly. 
Trust in government appears to predict higher depression symptoms. Further analyses indicated that trust in government and depression symptoms do not correlate, which is why the statistical significance has no topical relevance (see supplementary materials).Contrasted with previous studies with cancer patients and the general population, prior mental illness was no predictor of an increase of psychological burden $[13,18,38,50]$. At this point, it is important to mention that the assessment of mental illness did not differentiate between disorders, even though the spectrum of mental illnesses is broad. Besides, patients with prior mental illness might not have reported an increase in mental health burden, as it already existed before the pandemic.

\subsection{Limitations}

A strong point of this study is its time of assessment, as it was conducted shortly after the first lock-down in Germany, which can be considered an early stage of the pandemic. Nevertheless, it might also be essential to assess the impact of the pandemic on mental health of diabetes patients at a different stage of the pandemic, as recent literature has shown that psychological burden and COVID-19-related fear varies between different periods of the pandemic [51,52]. Hence, the time of study participation might impact self-reported data. As the analysed sample of the present study was too small and the survey period too short, it was not possible to assess the impacts of COVID-19 at different periods of the pandemic. Further limitations must be considered: As the data of the survey was collected via online and analogue channels, a possible selection bias cannot be ruled out. Since more participants in our sample were diagnosed with diabetes type 1, it must be questioned whether our group of participants is sufficiently representative of diabetes patients world-wide, as the majority of affected patients suffer from type 2 [53]. Also, recent literature shows that patients with type 2 diabetes had almost twice the prevalence of depression symptoms compared to patients with type 1 diabetes [54]. Further analyses of our data are in line with this observation (see supplementary materials). As the data is based on selfreport, an objective verification of the diabetes diagnosis is not possible. In addition to this, more women participated in the present study, which should also be considered. The fact that non-responders could not be identified due to the anonymous approach of data assessment, adds to the aforementioned selection bias. Moreover, the results are not based on longitudinal, but on cross-sectional data. Hence, causality cannot be assumed. The instruments used to collect data regarding mental health and health status prior to the onset of COVID-19, were not validated as no such instruments existed at the time the study was designed and launched. To cope with this limitation, all instruments to investigate mental health and health status in the present study were adjusted to assess mental health before the COVID-19 outbreak retrospectively. Hence, the occurrence of recall-biased assessments should be considered. Finally, it must be critically noted that determinants of change in mental health are still not clear and that reasons for mental health impairment can differ in individuals. A proper control group would be needed to make generalizable observations in terms of distinct mental health impairments that are actually COVID19-related. Consequently, it is essential not to assume all measured effects to be caused by COVID-19. Despite of the limitations, this study provides a practicable approach to investigate mental health changes shortly after the sudden onset of the virus outbreak. Further research is recommended and should consider the abovementioned limitations while conceptualizing all instruments used.

Page $14 / 22$ 


\section{Conclusions}

In summary, this study shows that German patients with diabetes reported increased mental health burden and impairment in health status since the COVID-19 outbreak. COVID-19-related fear appears to play an important role in diabetes patients as it was associated with increased depression symptoms, generalized anxiety symptoms, and distress. Hence, our findings could be helpful in the effort to improve the efficacy of psychological support strategies for diabetes patients suffering from the ongoing pandemic and their increased risk of a potentially severe COVID-19 course. As the crisis continues, further research is needed to assess possible causes of mental health impairment during a pandemic and potential protective factors in order to support the development of preventive treatments for individuals diagnosed with diabetes.

\section{Abbreviations}

COVID-19 = Corona Virus Disease-19

DT = distress thermometer

EQ-5D-3L = European Quality of Life 5 Dimensions 3 Level questionnaire

$\mathrm{GAD}=$ generalized anxiety disorder

$\mathrm{PHQ}=$ patient health questionnaire

SARS-CoV-2 = Severe acute respiratory syndrome coronavirus type 2

\section{Declarations}

Ethics approval and consent to participate: The study was conducted according to the guidelines of the Declaration of Helsinki, and approved by the Ethics Committees of the University Hospital Essen (209307-BO). All participants gave electronic written informed consent.

Consent for publication: All participants gave electronic written informed consent.

Availability of data and materials: The data-sets used and analysed during the current study available from the corresponding author on reasonable request.

Competing interests: The authors declare that they have no competing interests.

Funding: This study was supported by the Essen University Medicine Foundation. The funder had no role in the design and conduct of the study; management, collection, analysis and interpretation of the data; preparation, review or approval of the manuscript and decision to submit the manuscript for publication. 
Author's contributions: S.M. and M.T. made substantial contributions to the study's design, actively participated in acquisition of data, statistical analysis, and interpretation of data, and prepared the manuscript. L.J., A.S., B.W., M.F., H.K. and V.M. actively participated in the interpretation of data and edited the manuscript. N.D., and S.T. made substantial contributions to the study's conception and revised the manuscript critically. A.B. and E.-M.S. made substantial contributions to the study's conception and design, actively participated in the interpretation of data, and revised the manuscript critically for important intellectual content.

Acknowledgements: Not applicable

\section{References}

1. Cucinotta D, Vanelli M. WHO Declares COVID-19 a Pandemic. Acta Biomed. 2020;91(1):157-60. 10.23750/abm.v91i1.9397.

2. Feehan J, Apostolopoulos V. Is COVID-19 the worst pandemic? Maturitas. 2021. 10.1016/j.maturitas.2021.02.001.

3. World Health Organization. WHO Coronavirus (COVID-19) Dashboard. Available from: https://covid19.who.int/. Acessed 1 May 2021.

4. Bedford J, Enria D, Giesecke J, Heymann DL, Ihekweazu C, Kobinger G, et al. COVID-19: towards controlling of a pandemic. The Lancet. 2020;395(10229):1015-8. 10.1016/s0140-6736(20)30673-5.

5. Garfin DR, Silver RC, Holman EA. The novel coronavirus (COVID-2019) outbreak: Amplification of public health consequences by media exposure. Health Psychol. 2020;39(5):355-7. 10.1037/hea0000875.

6. Lima CKT, Carvalho PMM, Lima I, Nunes J, Saraiva JS, de Souza RI, et al. The emotional impact of Coronavirus 2019-nCoV (new Coronavirus disease). Psychiatry Res. 2020;287:112915. 10.1016/j.psychres.2020.112915.

7. Casagrande M, Favieri F, Tambelli R, Forte G. The enemy who sealed the world: effects quarantine due to the COVID-19 on sleep quality, anxiety, and psychological distress in the Italian population. Sleep Med. 2020;75:12-20. 10.1016/j.sleep.2020.05.011.

8. Choi EPH, Hui BPH, Wan EYF. Depression and Anxiety in Hong Kong during COVID-19. Int J Environ Res Public Health. 2020;17(10). 10.3390/ijerph17103740.

9. Huang Y, Zhao N. Generalized anxiety disorder, depressive symptoms and sleep quality during COVID-19 outbreak in China: a web-based cross-sectional survey. Psychiatry Res. 2020;288:112954. 10.1016/j.psychres.2020.112954.

10. Rahman MA, Hoque N, Alif SM, Salehin M, Islam SMS, Banik B, et al. Factors associated with psychological distress, fear and coping strategies during the COVID-19 pandemic in Australia. Global Health. 2020;16(1):95. 10.1186/s12992-020-00624-w.

11. Rajkumar RP. COVID-19 and mental health: A review of the existing literature. Asian J Psychiatr. 2020;52:102066. 10.1016/j.ajp.2020.102066. 
12. Wang C, Pan R, Wan X, Tan Y, Xu L, Ho CS, et al. Immediate Psychological Responses and Associated Factors during the Initial Stage of the 2019 Coronavirus Disease (COVID-19) Epidemic among the General Population in China. Int J Environ Res Public Health. 2020;17(5). 10.3390/ijerph17051729.

13. Bäuerle A, Steinbach J, Schweda A, Beckord J, Hetkamp M, Weismüller B, et al. Mental Health Burden of the COVID-19 Outbreak in Germany: Predictors of Mental Health Impairment. J Prim Care Community Health. 2020;11:2150132720953682. 10.1177/2150132720953682.

14. Skoda EM, Bäuerle A, Schweda A, Dörrie N, Musche V, Hetkamp M, et al. Severely increased generalized anxiety, but not COVID-19-related fear in individuals with mental illnesses: A population based cross-sectional study in Germany. Int J Soc Psychiatry. 2020:20764020960773. $10.1177 / 0020764020960773$.

15. Gomez-Salgado J, Andres-Villas M, Dominguez-Salas S, Diaz-Milanes D, Ruiz-Frutos C. Related Health Factors of Psychological Distress During the COVID-19 Pandemic in Spain. Int J Environ Res Public Health. 2020;17(11). 10.3390/ijerph17113947.

16. Solomou I, Constantinidou F. Prevalence and Predictors of Anxiety and Depression Symptoms during the COVID-19 Pandemic and Compliance with Precautionary Measures: Age and Sex Matter. Int J Environ Res Public Health. 2020;17(14). 10.3390/ijerph17144924.

17. Kohler H, Bäuerle A, Schweda A, Weismüller B, Fink M, Musche V, et al. Increased COVID-19-related fear and subjective risk perception regarding COVID-19 affects behavior in individuals with internal high-risk diseases. J Prim Care Community Health. 2021;12:2150132721996898.

$10.1177 / 2150132721996898$.

18. Wang Y, Duan Z, Ma Z, Mao Y, Li X, Wilson A, et al. Epidemiology of mental health problems among patients with cancer during COVID-19 pandemic. Transl Psychiatry. 2020;10(1):263.

10.1038/s41398-020-00950-y.

19. Yang J, Zheng Y, Gou X, Pu K, Chen Z, Guo Q, et al. Prevalence of comorbidities and its effects in patients infected with SARS-CoV-2: a systematic review and meta-analysis. Int $\mathrm{J}$ Infect Dis. 2020;94:91-5. 10.1016/j.ijid.2020.03.017.

20. Guan WJ, Ni ZY, Hu Y, Liang WH, Ou CQ, He JX, et al. Clinical Characteristics of Coronavirus Disease 2019 in China. N Engl J Med. 2020;382(18):1708-20. 10.1056/NEJMoa2002032.

21. Harrison SL, Fazio-Eynullayeva E, Lane DA, Underhill P, Lip GYH. Comorbidities associated with mortality in 31,461 adults with COVID-19 in the United States: A federated electronic medical record analysis. PLoS Med. 2020;17(9):e1003321. 10.1371/journal.pmed.1003321.

22. Cariou B, Hadjadj S, Wargny M, Pichelin M, Al-Salameh A, Allix I, et al. Phenotypic characteristics and prognosis of inpatients with COVID-19 and diabetes: the CORONADO study. Diabetologia. 2020;63(8):1500-15. 10.1007/s00125-020-05180-x.

23. World Health Organization. Health Topics: Diabetes. Available from: https://www.who.int/healthtopics/diabetes\#tab=tab_1. Acessed 1 May 2021.

24. Roncon L, Zuin M, Rigatelli G, Zuliani G. Diabetic patients with COVID-19 infection are at higher risk of ICU admission and poor short-term outcome. J Clin Virol. 2020;127:104354. 
10.1016/j.jcv.2020.104354.

25. Saha S, Al-Rifai RH, Saha S. Diabetes prevalence and mortality in COVID-19 patients: a systematic review, meta-analysis, and meta-regression. J Diabetes Metab Disord. 2021:1-12. 10.1007/s40200021-00779-2.

26. Pal R, Bhadada SK. COVID-19 and diabetes mellitus: An unholy interaction of two pandemics. Diabetes Metab Syndr. 2020;14(4):513-7. 10.1016/j.dsx.2020.04.049.

27. Weismüller B, Schweda A, Dörrie N, Musche V, Fink M, Kohler H, et al. Different Correlates of COVID19-Related Adherent and Dysfunctional Safety Behavior. Front Public Health. 2020;8:625664. 10.3389/fpubh.2020.625664.

28. Musche V, Kohler H, Bäuerle A, Schweda A, Weismüller B, Fink M, et al. COVID-19-Related Fear, Risk Perception, and Safety Behavior in Individuals with Diabetes. Healthcare (Basel). 2021;9(4). 10.3390/healthcare9040480.

29. Joensen LE, Madsen KP, Holm L, Nielsen KA, Rod MH, Petersen AA, et al. Diabetes and COVID-19: psychosocial consequences of the COVID-19 pandemic in people with diabetes in Denmark-what characterizes people with high levels of COVID-19-related worries? Diabet Med. 2020;37(7):1146-54. 10.1111/dme.14319.

30. Forte G, Favieri F, Tambelli R, Casagrande M. The Enemy Which Sealed the World: Effects of COVID19 Diffusion on the Psychological State of the Italian Population. J Clin Med. 2020;9(6). 10.3390/jcm9061802.

31. Turner J, Kelly B. Emotional dimensions of chronic disease. West J Med. 2000;172(2):124-8. 10.1136/ewjm.172.2.124.

32. Rubin RR, Peyrot M. Quality of life and diabetes. Diabetes/Metabolism Research and Reviews. 1999;15(3):205-18. 10.1002/(sici)1520-7560(199905/06)15:3 < 205::Aid-dmrr29 > 3.0.Co;2-o.

33. Rubin RR, Peyrot M. Psychological issues and treatments for people with diabetes. J Clin Psychol. 2001;57(4):457-78. 10.1002/jclp.1041.

34. Ali S, Stone MA, Peters JL, Davies MJ, Khunti K. The prevalence of co-morbid depression in adults with Type 2 diabetes: a systematic review and meta-analysis. Diabet Med. 2006;23(11):1165-73. 10.1111/j.1464-5491.2006.01943.x.

35. Grigsby AB, Anderson RJ, Freedland KE, Clouse RE, Lustman PJ. Prevalence of anxiety in adults with diabetes. Journal of Psychosomatic Research. 2002;53(6):1053-60. 10.1016/s00223999(02)00417-8.

36. Tan KC, Chan GC, Eric H, Maria Al, Norliza MJ, Oun BH, et al. Depression, anxiety and stress among patients with diabetes in primary care: A cross-sectional study. Malays Fam Physician. 2015;10(2):9-21.

37. Gonzalez JS, Tanenbaum ML, Commissariat PV. Psychosocial factors in medication adherence and diabetes self-management: Implications for research and practice. Am Psychol. 2016;71(7):539-51. $10.1037 / \mathrm{a} 0040388$. 
38. Bäuerle A, Musche V, Schmidt K, Schweda A, Fink M, Weismüller B, et al. Mental Health Burden of German Cancer Patients before and after the Outbreak of COVID-19: Predictors of Mental Health Impairment. Int J Environ Res Public Health. 2021;18(5). 10.3390/ijerph18052318.

39. Kroenke K, Spitzer RL, Williams JB. The Patient Health Questionnaire-2: validity of a two-item depression screener. Med Care. 2003;41(11):1284-92. 10.1097/01.MLR.0000093487.78664.3C.

40. Kroenke K, Spitzer RL, Williams JB, Monahan PO, Löwe B. Anxiety disorders in primary care: prevalence, impairment, comorbidity, and detection. Ann Intern Med. 2007;146(5):317-25. 10.7326/0003-4819-146-5-200703060-00004.

41. Spitzer RL, Kroenke K, Williams JB, Löwe B. A brief measure for assessing generalized anxiety disorder: the GAD-7. Arch Intern Med. 2006;166(10):1092-7. 10.1001/archinte.166.10.1092.

42. Hinz A, Klein AM, Brahler E, Glaesmer H, Luck T, Riedel-Heller SG, et al. Psychometric evaluation of the Generalized Anxiety Disorder Screener GAD-7, based on a large German general population sample. J Affect Disord. 2017;210:338-44. 10.1016/j.jad.2016.12.012.

43. Brooks R. EuroQol: the current state of play. Health Policy. 1996;37(1):53-72. 10.1016/01688510(96)00822-6.

44. Schmidt AF, Finan C. Linear regression and the normality assumption. J Clin Epidemiol. 2018;98:146-51. 10.1016/j.jclinepi.2017.12.006.

45. Bäuerle A, Skoda EM, Dorrie N, Böttcher J, Teufel M. Psychological support in times of COVID-19: the Essen community-based CoPE concept. J Public Health (Oxf). 2020;42(3):649-50. 10.1093/pubmed/fdaa053.

46. Weiner L, Berna F, Nourry N, Severac F, Vidailhet P, Mengin AC. Efficacy of an online cognitive behavioral therapy program developed for healthcare workers during the COVID-19 pandemic: the REduction of STress (REST) study protocol for a randomized controlled trial. Trials. 2020;21(1):870. 10.1186/s13063-020-04772-7.

47. Gústavsson SM, Salkovskis PM, Sigurðsson JF. Cognitive analysis of specific threat beliefs and safety-seeking behaviours in generalised anxiety disorder: revisiting the cognitive theory of anxiety disorders. Behavioural and Cognitive Psychotherapy. 2021:1-14. 10.1017/s135246582100014x.

48. Dong L, Hu S, Gao J. Discovering drugs to treat coronavirus disease 2019 (COVID-19). Drug Discov Ther. 2020;14(1):58-60. 10.5582/ddt.2020.01012.

49. Wang T, Du Z, Zhu F, Cao Z, An Y, Gao Y, et al. Comorbidities and multi-organ injuries in the treatment of COVID-19. The Lancet. 2020;395(10228). 10.1016/s0140-6736(20)30558-4.

50. Yao H, Chen J-H, Xu Y-F. Patients with mental health disorders in the COVID-19 epidemic. The Lancet Psychiatry. 2020;7(4). 10.1016/s2215-0366(20)30090-0.

51. Hetkamp M, Schweda A, Bäuerle A, Weismüller B, Kohler H, Musche V, et al. Sleep disturbances, fear, and generalized anxiety during the COVID-19 shut down phase in Germany: relation to infection rates, deaths, and German stock index DAX. Sleep Med. 2020;75:350-3.

10.1016/j.sleep.2020.08.033. 
52. Skoda EM, Spura A, De Bock F, Schweda A, Dörrie N, Fink M, et al. Change in psychological burden during the COVID-19 pandemic in Germany: fears, individual behavior, and the relevance of information and trust in governmental institutions. Bundesgesundheitsblatt Gesundheitsforschung Gesundheitsschutz. 2021;64(3):322-33. 10.1007/s00103-021-03278-0.

53. World Health Organization. Fact sheets: Diabetes. Available from: https://www.who.int/newsroom/fact-sheets/detail/diabetes. Acessed 1 May 2021.

54. Bak E, Marcisz-Dyla E, Mlynarska A, Sternal D, Kadlubowska M, Marcisz C. Prevalence of Depressive Symptoms in Patients with Type 1 and 2 Diabetes Mellitus. Patient Prefer Adherence. 2020;14:44354. 10.2147/PPA.S237767.

\section{Figures}


a) Depression symptoms

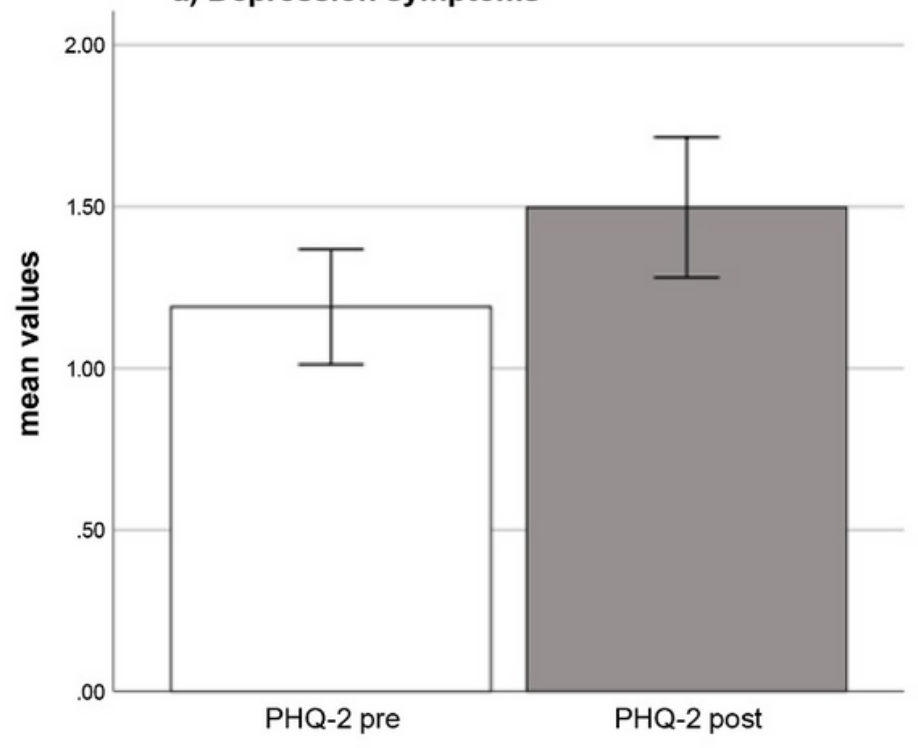

c) Distress

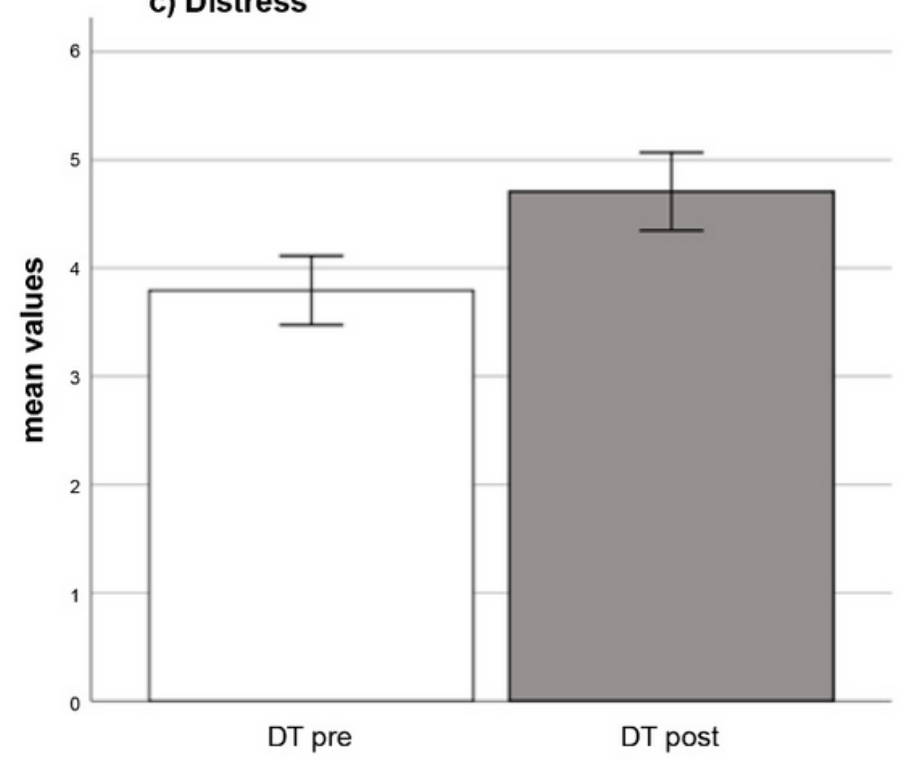

b) Generalized anxiety symptoms
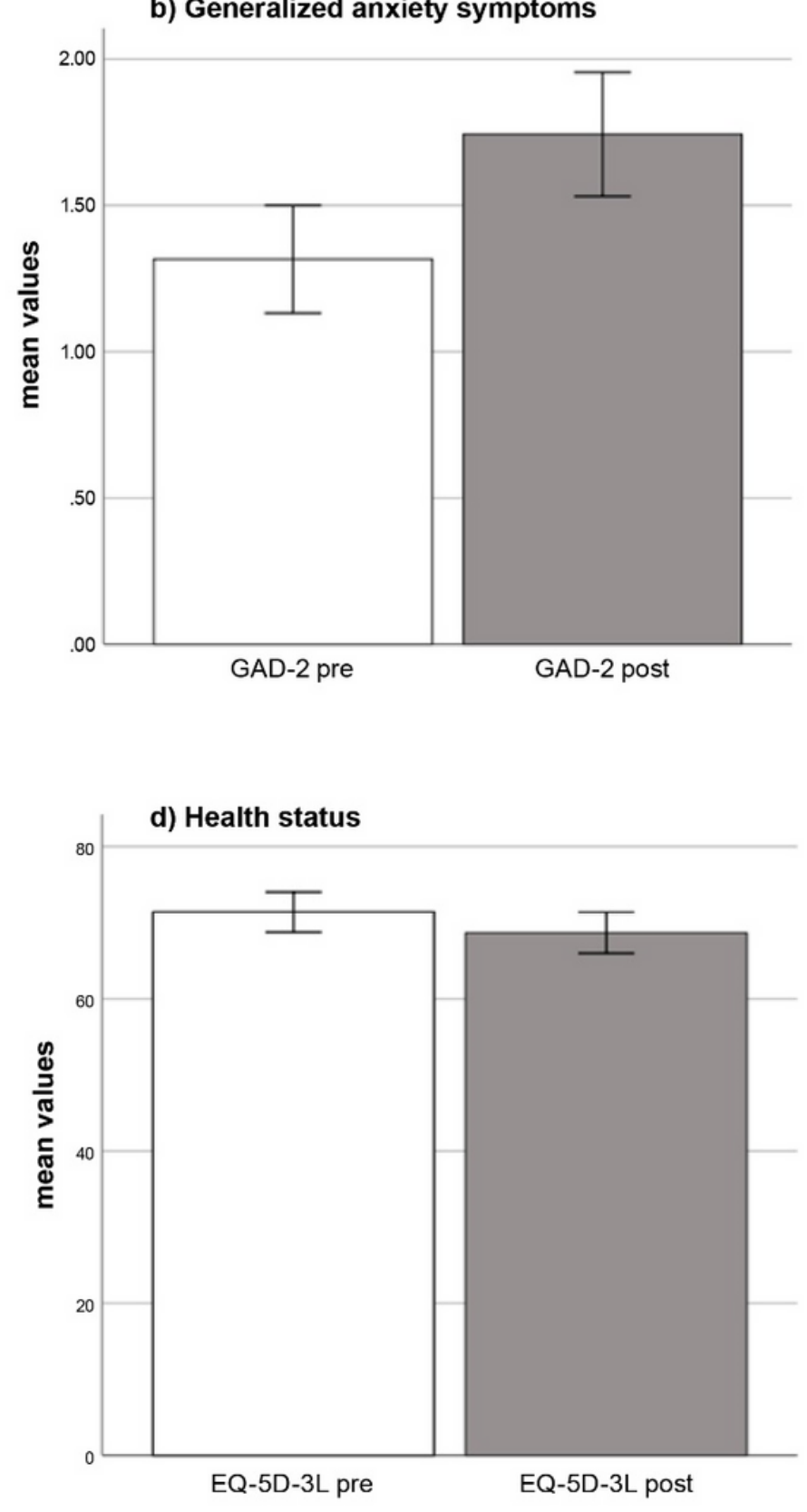

\section{Figure 1}

Mental health and health status mean scores before and after the COVID-19 outbreak. Mean values and $95 \% \mathrm{Cl}$ as error bars before (pre) and after (post) the outbreak of COVID-19 of PHQ-2: Patient Health Questionnaire-2, MPHQ-2 pre = 1.50 vs. MPHQ-2 post $=1.19$ (a); GAD-2: Generalized Anxiety Disorder Scale-2, MGAD-2 pre $=1.74$ vs. MGAD-2 post $=1.32$ (b); DT: Distress Thermometer, MDT pre $=4.71$ vs. MDT post $=3.79$ (c); EQ-5D-3L: European Quality of Life 5 Dimensions 3 Level, MEQ-5D-3L pre $=68.68$ vs. MEQ-5D-3L post $=71.40(\mathrm{~d})$.

\section{Supplementary Files}


This is a list of supplementary files associated with this preprint. Click to download.

- Moradianetalsupplementarymaterials.docx 